\title{
Nanotechnology to Improve the Performances of Hydrodynamic Surfaces
}

\author{
Ali Alshehri 1,** Philippe Champagne ${ }^{2}$, Laurent Keirsbulck ${ }^{3}$ and El Hadj Dogheche ${ }^{1}$ \\ 1 IEMN-DOAE, Univ. Polytechnique Hauts-de-France, F-59313 Valenciennes, France; \\ elhadj.dogheche@uphf.fr \\ 2 LMCPA, Univ. Polytechnique Hauts-de-France, F-59600 Maubeuge, France ; philippe.champagne@uphf.fr \\ 3 LAMIH, Univ. Polytechnique Hauts-de-France, F-59313 Valenciennes, France ; laurent.keirsbulck@uphf.fr \\ * Correspondence: ali.alshehri@etu.uphf.fr; Tel.: +33 762337825
}

Received: 11 February 2019; Accepted: 21 March 2019; Published: 21 March 2019

\begin{abstract}
The nature continues to inspire scientists to adapt solutions in order to satisfy the human needs and attain unreachable performances with new technologies. In this study, the superhydrophobicity of the Lotus leaf is our source of inspiration. The duplication of this natural phenomenon may enhance the maritime metallic surfaces corrosion and its mechanical friction. In our case, we are investigating super-hydrophobic maritime surfaces using a simple, low cost and scalable coating method. A hydrothermal method is used to create zinc oxide ( $\mathrm{ZnO}$ ) nanorods (NRs) and an evaporation method to apply the Octadecyltrimethoxysilane (ODS). At the end, the superhydrophobic surface (SHS) is obtained on a maritime aluminum substrate coated by a commercial epoxy paint. The characterization of our SHS gives high water contact angle (WCA) and small sliding angle (SA) of water droplets on the treated surface. We have raised the WCA of the epoxy painted aluminum surface from $98^{\circ}$ to more than $152^{\circ}$ and reduce the SA of $46^{\circ}$ to lower than $7^{\circ}$. We have also studied the sliding speed (SS) that have been largely raised from $0.04 \mathrm{~m} \mathrm{~s}^{-1}$ in the epoxy case to $1.3 \mathrm{~m} \mathrm{~s}^{-1}$ after treatment.
\end{abstract}

Keywords: super-hydrophobic surfaces; nanotechnology; nanorods; $\mathrm{ZnO}$; water contact angle; sliding angle; sliding speed; hydrodynamic surfaces; epoxy paint

\section{Introduction}

The different surface reactions against deposed water drops are known as the surface hydrophobicity or wettability. It has been a very interesting subject for the Scientifics to study. Young in 1805 [1] described the water contact angle $(\theta)$ as a relation of surface energy $(\gamma)$ interaction between the 3 contacting surfaces; liquid (l), solid (s) and gas (g) when a drop of water is laid on a solid surface:

$$
\cos \left(\theta_{\mathrm{y}}\right)=\frac{\gamma_{\mathrm{sg}}-\gamma_{\mathrm{sl}}}{\gamma_{\mathrm{lg}}}
$$

Wenzel in 1936 [2] introduced the surface roughness $(r)$ effect on Young Equation (1).

$$
\cos \left(\theta_{\mathrm{W}}\right)=\mathrm{r} \cos \left(\theta_{\mathrm{y}}\right)
$$

Furthermore in 1944, Cassie and Baxter [3] introduced the area fraction $(f)$ in Wenzel model by studying the effect of trapped air pockets in porous surfaces.

$$
\cos \left(\theta_{\mathrm{CB}}\right)=f\left(\cos \theta_{\mathrm{y}}+1\right)-1
$$

It is well known that $\mathrm{ZnO}$ nanomaterials are frequently employed for their antibacterial capacities in a very wide field of applications [4,5]. ZnO NRs material has also confirmed its 
antimicrobial capacity in the paint experiences [6] and in other applications like textile [7], The embedment of ZnO NRs in epoxy coat has improved anti-corrosion and hydrophobic properties with WCA of $128^{\circ}$ [8]. The epoxy coat blended with $\mathrm{ZnO}$ nanorods has proven a very good corrosion resistance capacity [9-12]. The addition of $\mathrm{ZnO}$ nanoparticles (NPs) in epoxy paint improves the adhesion and the lifetime of the coat [11]. In our study, we investigate the super hydrophobicity of treated $\mathrm{ZnO}$ NRs and the dynamic gain in the sliding speed on an epoxy painted maritime aluminum disc.

\section{Materials and Methods}

\subsection{Paint Application}

We used an aluminum disc of $100 \mathrm{~mm}$ diameter as substrate; a maritime applications aluminum alloy 6061 from Goodfellow (Lille, France). Furthermore, this aluminum substrate is coated by an epoxy paint applied by Damen Shipyards Dunkerque (Dunkerque, France) throw two layer of commercial paints by means of airless spray. A primer layer of $40 \mu \mathrm{m}$ thickness (Intergard 269, International) is used to prepare the surface before having $250 \mu \mathrm{m}$ thickness epoxy layer (Interzone 954, International). A curing period of 5 days in an atmospheric temperature is respected before the next step.

\subsection{Super-Hydrophobic Surface Creation}

In order to test our solution on an industrial coat, we deployed two steps to implement the $\mathrm{ZnO}$ NRs by hydrothermal method on the painted substrate; a first step is deployed to have the $\mathrm{ZnO}$ seeds, the second one is to grow the $\mathrm{ZnO} N R$.

\subsection{1. $\mathrm{ZnO}$ Seeds Synthesis}

The Seeds are prepared with zinc acetate dihydrate (Fisher, Hampton, NH, USA) and granulated sodium hydroxide (98\%, Alfa Aesar, Haverhill, MA, USA) in absolute methanol (Fisher) as follow:

- Solution A is obtained by dissolving $90 \mathrm{mmol} / \mathrm{L}$ of zinc acetate dihydrate in methanol.

- Solution B is obtained by dissolving $75 \mathrm{mmol} / \mathrm{L}$ of sodium hydroxide in methanol.

- Solution C is obtained by slowly addition of Solution B (1 drop/s) to the Solution A while stirring at $60^{\circ} \mathrm{C}$. At the end, a transparent seed solution is obtained.

After $3 \mathrm{~h}$ of stirring at room temperature, the seeds Solution $\mathrm{C}$ is ready to immerse the sample. The sample is immersed for $5 \mathrm{~min}$ in the solution while stirring. Then, the sample is dried at $90{ }^{\circ} \mathrm{C}$ for $20 \mathrm{~min}$. This process is repeated 5 times to ensure the uniform seeds application onto the sample. Finally, the sample is annealed at $90^{\circ} \mathrm{C}$ for $30 \mathrm{~min}$. Figure 1 illustrates the seeds preparation protocol.

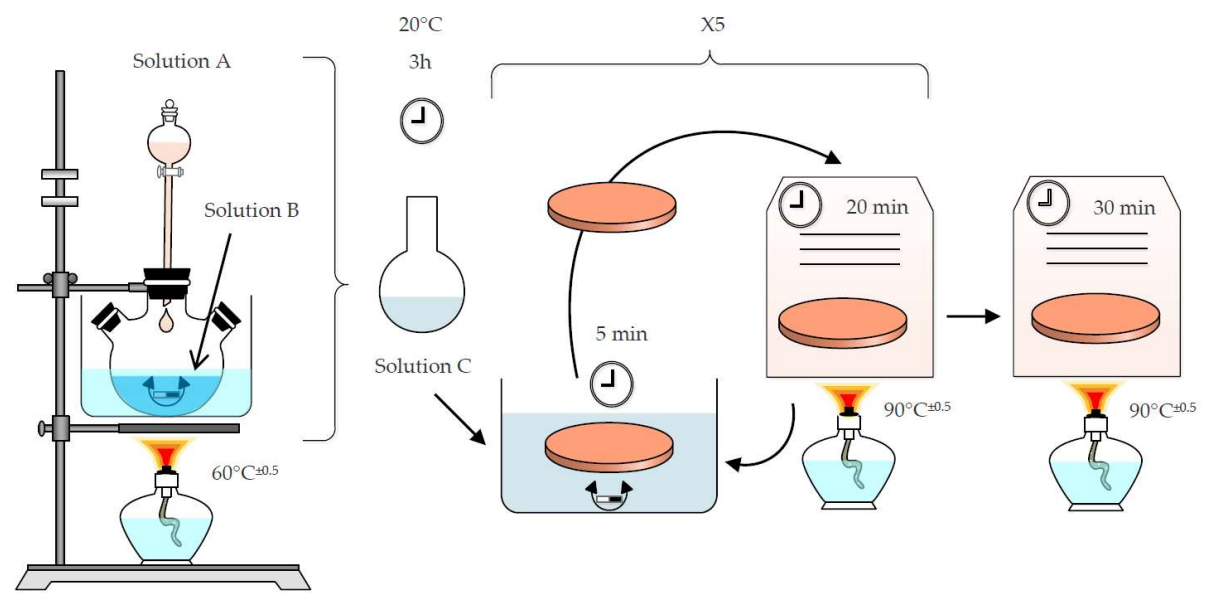

Figure 1. Seeds preparation process. 


\subsection{2. $\mathrm{ZnO}$ Nanorods synthesis}

In the second step, nanorods are grown by hydrothermal method by adding $100 \mathrm{mmol} / \mathrm{L}$ of zinc nitrate hexahydrate (Extra Pure, SLR, Fisher) and $100 \mathrm{mmol} / \mathrm{L}$ hexamethylenetetramine (ACS, 99+\%, Alfa Aesar) solutions. $250 \mathrm{~mL}$ of each solution is added to a Teflon bottle containing the sample. The mixture is agitated at $90{ }^{\circ} \mathrm{C}$ for $24 \mathrm{~h}$. Then, the samples are washed in distilled water for 3 times and dried at $90^{\circ} \mathrm{C}$ for $30 \mathrm{~min}$. Figure 2 shows the $\mathrm{ZnO}$ nanorods process.
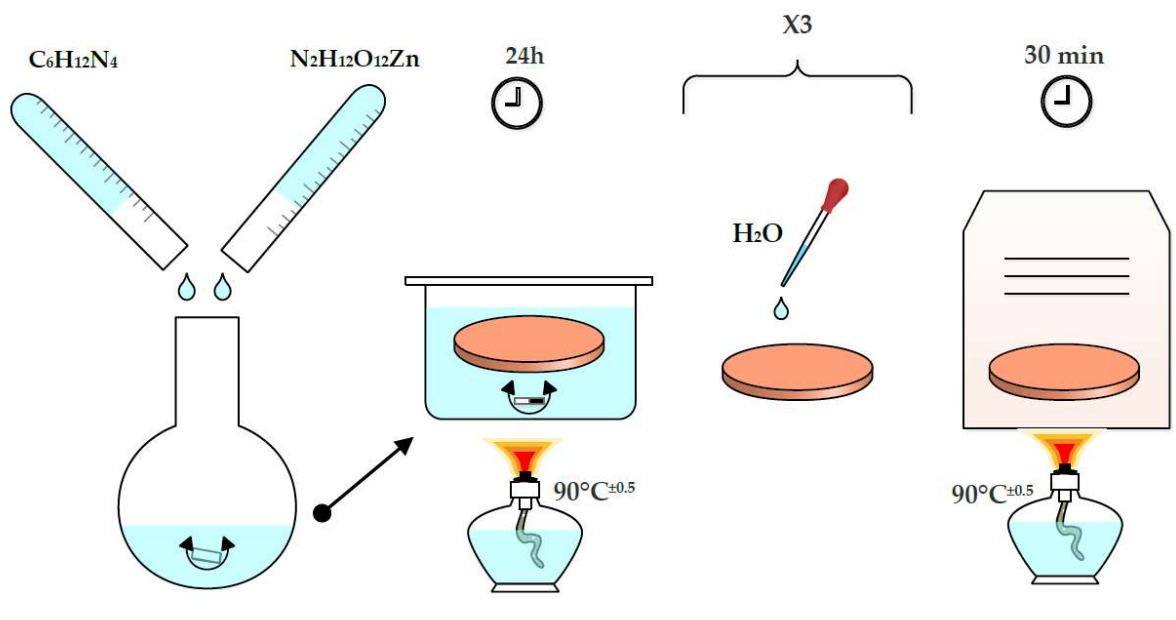

Figure 2. ZnO Nanorods preparation process.

\subsubsection{Octadecyltrimethoxysilane (ODS) Deposition}

A lower energy layer is necessary above the $\mathrm{ZnO}$ NRs. This layer is done by evaporation method. $200 \mu \mathrm{L}$ of Octadecyltrimethoxysilane (90\%, Tech., ACROS Organics, Leicestershire, UK) is placed in the bottom of Teflon bottle with the sample holding horizontally for $6 \mathrm{~h}$ at $90{ }^{\circ} \mathrm{C}$.

\section{Characterizations}

\subsection{Morphology Properties}

The microscopic analysis of the $\mathrm{ZnO}$ NRs raised on the epoxy painted aluminum disc are done by the scanning electronic microscopy SEM (ZEISS ULTRA 55 and ZEISS ULTRA 55VP, Jena, Germany). The surfaces morphologies observations are done by Microscope confocal (Leica DCM 3D, Leica Microsystems, Wizlar, Germany).

\subsection{Hydrophobic Properties}

The sample is characterized to determine the improvement in the water contact angle (WCA) and the sliding angle (SA) values throw the three surface states: (1) aluminum substrate only; (2) the substrate coated by the epoxy paint; and (3) the epoxy painted sample treated by $\mathrm{ZnO}+$ ODS.

WCA and SA are measured by the means of a drop shape analyzer (DSA25S, Kruss, Hamburg, Germany). A drop of distillate water of $2 \mu \mathrm{L}$ is posed on the surface with a debit of $2.67 \mu \mathrm{L} / \mathrm{s}$, the drop is then observed by an integrated camera (TIS DFK 37BUX273, The Imaging Source, Bremen, Germany) to trace the surface line and the drop shape line, a Young Laplace analyses model is selected to determine the WCA. Ten measurements are performed on each type of surface. The SA is measured by the same system employing an automated tilt table with an inclining speed of $0.5^{\circ} \mathrm{s}$. Ten measurements also are performed on each type of surface. 


\subsection{Hydrodynamic Properties}

To measure the sliding speed (SS), the aluminum disc is hold vertically and a drop of distillate water is released freely on the top of the vertical disc side. The droplet movement is tracked by a high speed camera (Phantom, M310, Vision Research, Wayne, NJ, USA) fitted with a $105 \mathrm{~mm}$ objective (AF-S VR Micro-Nikkor, Nikon, Tokyo, Japan) and Phantom MultiCam Application software is used to control the drop traveled distance and to find out the frequencies of pictures. In deed, 1000 pictures per second are taken and treated to determine the drop sliding speed. The measurements are done between each two points respecting a constant traveled distance $(d=0.01 \mathrm{~m})$. To determine the time between two points, we converted their pictures frequencies' to time $(t)$ :

$$
t(s)=\frac{1}{1000} \times\left[\frac{1}{f q(t)}-\frac{1}{f q(t+\nabla t)}\right]
$$

To control the drop size and flow, a syringe pump (100, Legato) is used to deliver regular water drop of $50 \mu \mathrm{L}$ with $1 \mathrm{~mL} / \mathrm{min}$. We note that, 10 trials are performed for each surface type. Figure 3 shows the SS measurement protocol and 2 positions of a drop at two different moments.

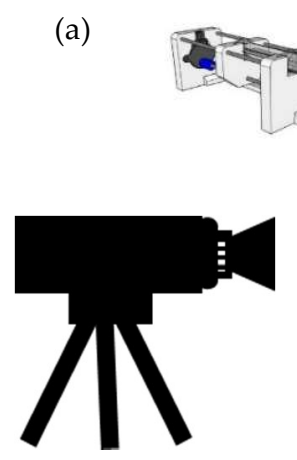

(b)

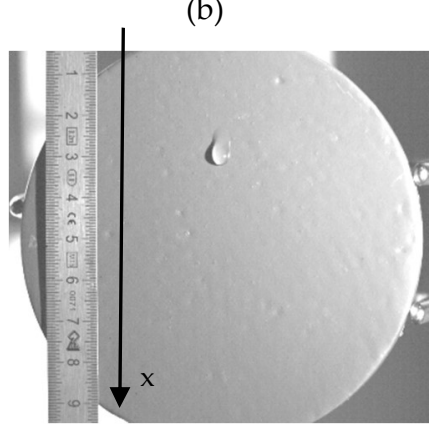

(c)

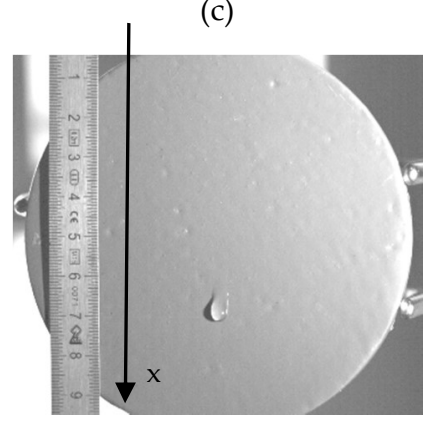

\section{NIIIIININ}

Figure 3. (a) Experimental set-up for Sliding Speed measurement; (b) Water drop image for the moment $(t)$; (c) Water drop image for the moment $(t+\Delta t)$.

\section{Results and Discussion}

\subsection{Surface Morphology}

The application of different layers on the aluminum substrate impacts the surface morphology. In our case, the surface roughness $\left(R_{\mathrm{a}}\right)$ of non treated aluminum substrate equals to $40 \mathrm{~nm}$. Howver, the application of the epoxy paint reduces it to $26 \mathrm{~nm}$. Finally, the application of our $\mathrm{ZnO}+$ ODS solution raise it to $58 \mathrm{~nm}$. This variation of surface roughness illustrated by Figures 4-6 corresponds to the different steps of evolution of treated alumina substrate targeting a lotus leaf nanostructure.

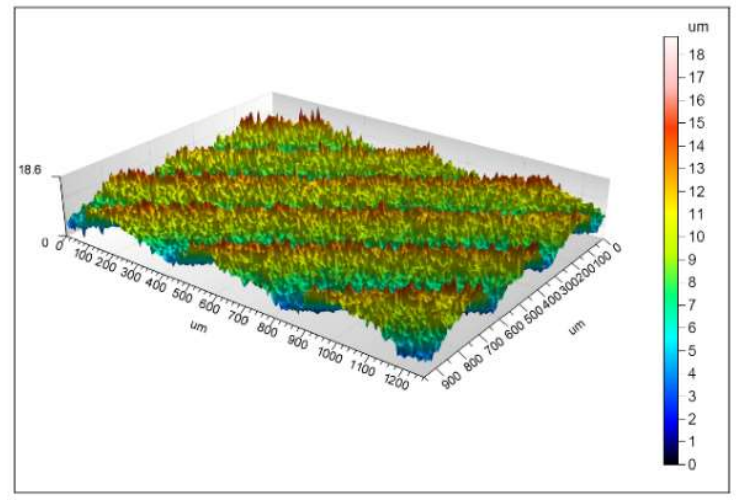

Figure 4. Aluminum surface morphology. $R_{\mathrm{a}}=40 \mathrm{~nm}$. 


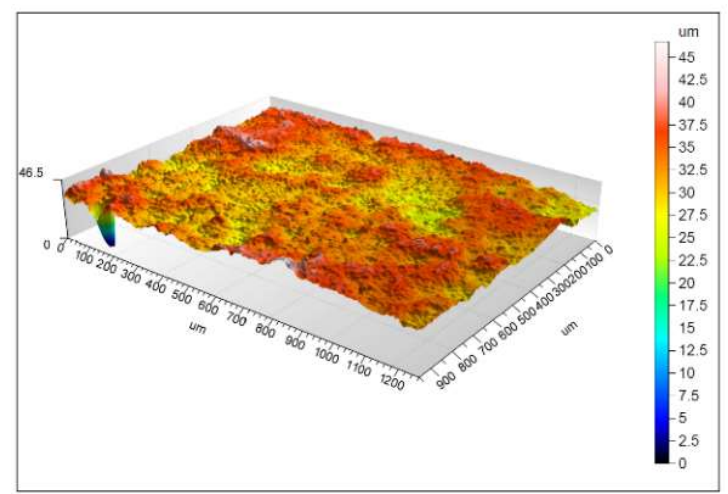

Figure 5. Aluminum surface + Epoxy paint morphology. $R_{\mathrm{a}}=26 \mathrm{~nm}$.

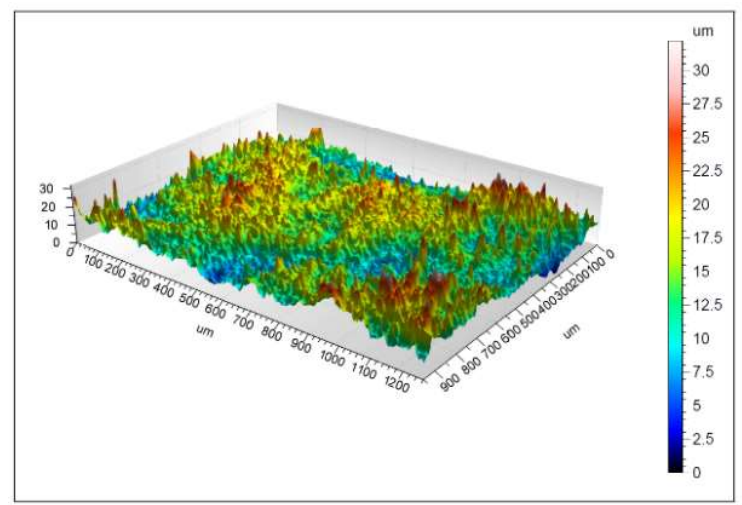

Figure 6. $\mathrm{ZnO}+$ ODS surface morphology. $R_{\mathrm{a}}=58 \mathrm{~nm}$.

\subsection{Nano Structural Results}

The obtained ZnO NRs on the epoxy layer after SEM analysis is shown in Figure 7. It has an oriented-vertically nanostructure with an average length of $300 \mathrm{~nm}$ and a diameter of $30 \mathrm{~nm}$. Obviously, the nanorods distribution is very dense with a great covering rate. This nanostructure helps to reduce the surface contact between water drops and the treated surface in order to create SHS beside the low energy layer of ODS.

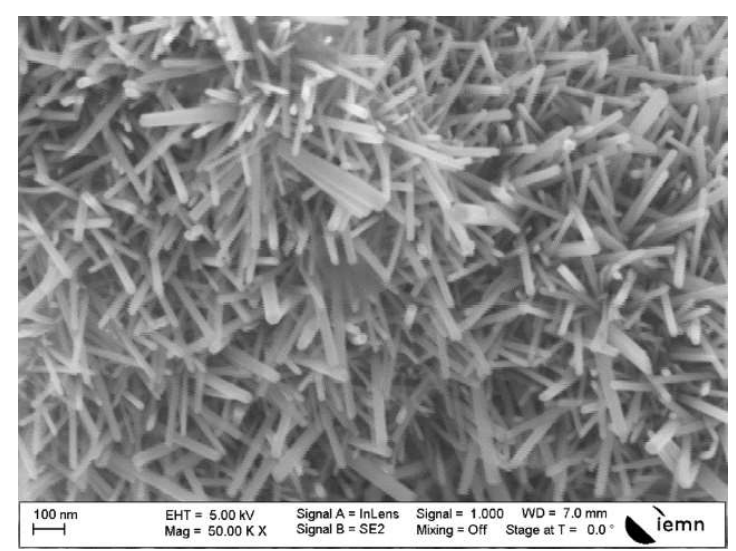

Figure 7. SEM analysis for $\mathrm{ZnO}$ nanorods deposited on aluminum substrate coated by epoxy paint.

\subsection{Wetting Results}

The WCA and SA values provide respectable indication of the surface wetting state. When a WCA $<90^{\circ}$ and $\mathrm{SA}>10^{\circ}$, the surface is considered hydrophilic. However, it is called hydrophobic when it has WCA $>90^{\circ} \& \mathrm{SA}<10^{\circ}$. Furthermore, a super-hydrophobic surface is attributed to the 
surfaces with $\mathrm{WCA}>150^{\circ}$ and $\mathrm{SA}<10^{\circ}$. According to the experimental results on our different surface states, Hydrophilic surfaces are obviously observed with the aluminum surface and the epoxy painted aluminum one. But the SHS is only appeared after the $\mathrm{ZnO}+$ ODS treatment. Table 1 summarizes the WCA \& SA throw the 3 stages of our treated aluminum surface.

Table 1. Water Contact Angle (WCA) and Sliding Angle (SA) of the aluminum substrate throw the 3 different surface states; (a) Not treated aluminum surface, (b) Epoxy painted surface, (c) Epoxy painted surface treated by $\mathrm{ZnO}+$ ODS and (d) Our target, the Super-hydrophobic Surface (SHS).

\begin{tabular}{ccc}
\hline Substrate & WCA $\left(^{\circ}\right)$ & SA $\left(^{\circ}\right)$ \\
\hline (a) Aluminum & 93 & 22 \\
(b) Epoxy & 97 & 46 \\
(c) ZnO + ODS & 152 & 7 \\
(d) SHS & 150 & 10 \\
\hline
\end{tabular}

\section{4. hydrodynamic Results}

The Sliding Speed (SS) measurement of the free vertical slipping drops on the aluminum disc shows the behavior of water droplet running the aluminum disc. Figure 8 shows the SS of $50 \mu \mathrm{L}$ water droplet running $100 \mathrm{~mm}$ distance for the 3 different surfaces. At the beginning, we observed low speed movement of water droplet on the non-treated aluminum substrate (SS max. $=0.25 \mathrm{~m} \mathrm{~s}^{-1}$ ). Coating the aluminum substrate with an epoxy paint will drive to lower speed (SS max. $=0.04 \mathrm{~m} \mathrm{~s}^{-1}$ ). But we have observed an important gain in the SS after having the $\mathrm{ZnO}+$ ODS treatment on our epoxy painted aluminum substrate with a SS max of $1.3 \mathrm{~m} \mathrm{~s}^{-1}$.

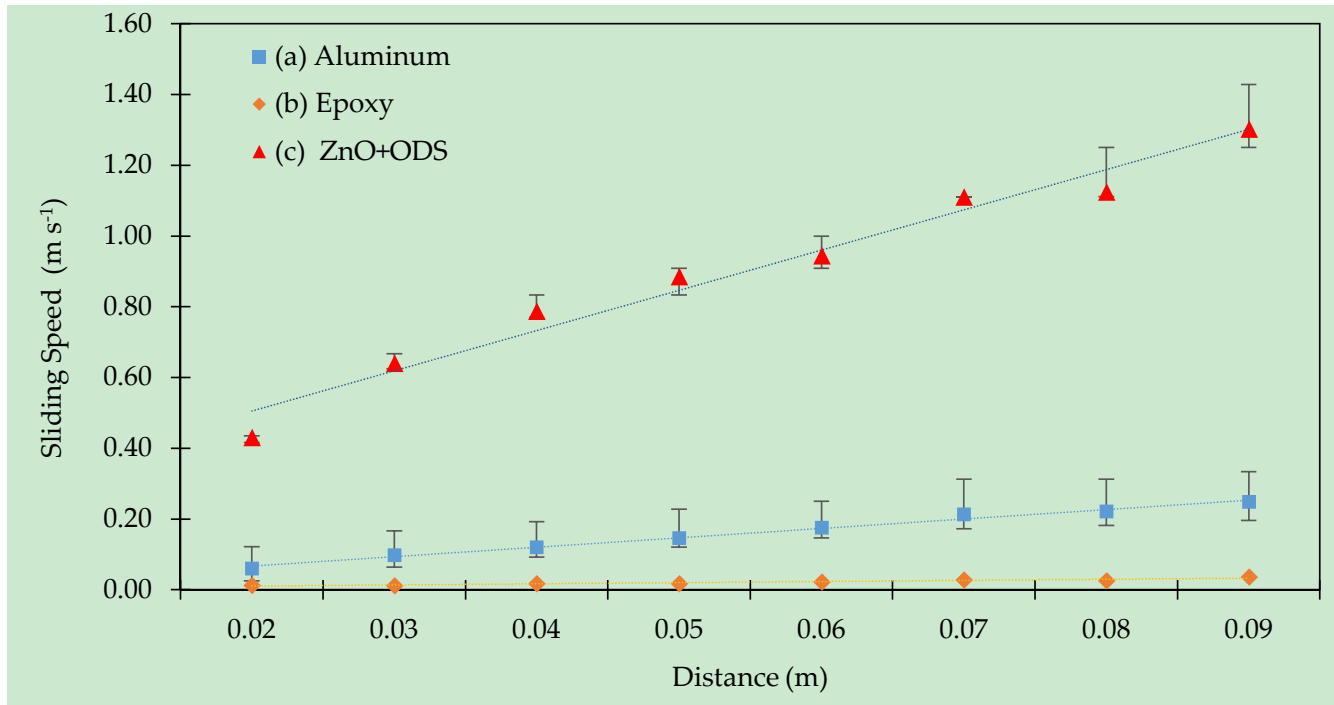

Figure 8. Sliding Speed (SS) measurement for 3 surface stages: (a) not treated aluminum surface; (b) epoxy painted surface; (c) epoxy painted surface treated by $\mathrm{ZnO}+$ ODS.

\section{Conclusions}

Nanorods are implemented successfully on an aluminum disc pre-coated by an industrial epoxy paint: the following geometry of $\mathrm{ZnO}$ NRs is $300 \mathrm{~nm}$ for length and $30 \mathrm{~nm}$ for diameter. The obtained NRs are covered by an ODS top layer in order to attain a super-hydrophobic surface. At the end, the SHS is obtained and characterized, a water contact angle WCA of more than $152^{\circ}$ and a sliding angle $\mathrm{SA}$ of less than $7^{\circ}$ are obtained (lotus leaf: $\mathrm{WCA}=150^{\circ} ; \mathrm{SA}=10^{\circ}$ ). The surface roughness is also raised from $26 \mathrm{~nm}$ in the epoxy case to $58 \mathrm{~nm}$ after treatment. The sliding speed SS progress is also investigated during the 3 different surface states; aluminum, painted aluminum and $\mathrm{ZnO}$ NRs treated painted aluminum. The SS is considerably raised from $0.04 \mathrm{~m} \mathrm{~s}^{-1}$ in the epoxy painted surface to more 
than $1.30 \mathrm{~m} \mathrm{~s}^{-1}$ in the $\mathrm{ZnO}$ NRs treated one. Those findings can be adapted to maritime surfaces and help to enhance the hydrodynamic performances to reduce mechanical friction and fuel consumption.

Acknowledgments: We would like to acknowledge Christophe Callewaert, Laurent Rembotte, Hubert Vincent and Damen Shipyards Dunkerque for the participation in substrate painting. We also wish to thank Sirehna Naval Group Nantes for helpful comments and discussions. This study was financially supported by Saudi Cultural Bureau in Paris.

Conflicts of interest: The authors declare no conflict of interest.

\section{References}

1. Thomas, Y.; Young, T. An essay on the cohesion of fluids. Philos. Trans. R. Soc. 1805, 95, 65-87.

2. Wenzel, R.N. Resistance of solid surfaces to wetting by water. J. Ind. Eng. Chem. 1936, 28, 988-994.

3. Cassie, B.D.; Baxter, S. Wettability Of porous surfaces. Trans. Faraday Soc. 1944, 40, 546-551.

4. Qi, K.; Cheng, B.; Yu, J.; Ho, W. Review on the improvement of the photocatalytic and antibacterial activities of ZnO. J. Alloy. Compd. 2017, 727, 792-820.

5. Sirelkhatim, A.; Mahmud, S.; Seeni, A.; Kaus, N.H.M.; Ann, L.C.; Bakhori, S.K.M.; Hasan, H; Mohamad, D. Review on zinc oxide nanoparticles: Antibacterial activity and toxicity mechanism. Nano-Micro Lett. 2015, 7, 219-242.

6. Hochmannova, L.; Vytrasova, J. Photocatalytic and antimicrobial effects of interior paints. Prog. Org. Coat. 2010, 67, 1-5.

7. Ashraf, M.; Dumont, F.; Campagne, C.; Champagne, P.; Perwuelz, A.; Leriche, A.; Chihib, N.E. Development of Antibacterial Polyester Fabric by Growth of ZnO Nanorods. J. Eng. Fiber. Fabr. 2014, 9, 1522.

8. Ammar, S.; Ramesh, K.; Vengadaesvaran, B.; Ramesh, S.; Arof, A.K. Amelioration of anticorrosion and hydrophobic properties of epoxy/PDMS composite coatings containing nano $\mathrm{ZnO}$ particles. Prog. Org. Coat. 2016, 92, 54-65.

9. Mostafaei, A.; Nasirpouri, F. Epoxy/polyaniline-ZnO nanorods hybrid nanocomposite coatings: Synthesis, characterization and corrosion protection performance of conducting paints. Prog. Org. Coat. 2014, 77, 146159.

10. Valença, D.P.; Alves, K.G.B.; Melo, C.P.D.; Bouchonneau, N. Study of the Efficiency of Polypyrrole/ZnO Nanocomposites as Additives in Anticorrosion Coatings. Mater. Res. 2015, 18, 273-278.

11. Shen, S.; Zuo, Y. The improved performance of Mg-rich epoxy primer on AZ91D magnesium alloy by addition of ZnO. Corros. Sci. 2014, 87, 167-178. 